\title{
A management algorithm for patients with intracranial pressure monitoring: the Seattle International Severe Traumatic Brain Injury Consensus Conference (SIBICC)
}

Gregory W. J. Hawryluk ${ }^{1}$, Sergio Aguilera ${ }^{2,3}$, Andras Buki ${ }^{4,5}$, Eileen Bulger ${ }^{6}$, Giuseppe Citerio ${ }^{7,8}$, D. Jamie Cooper ${ }^{9,10}$, Ramon Diaz Arrastia ${ }^{11}$, Michael Diringer ${ }^{12,13}$, Anthony Figaji ${ }^{14}$, Guoyi Gao ${ }^{15}$, Romergryko Geocadin ${ }^{16}$, Jamshid Ghajar ${ }^{17}$, Odette Harris ${ }^{18}$, Alan Hoffer ${ }^{19}$, Peter Hutchinson ${ }^{20}$, Mathew Joseph ${ }^{21}$, Ryan Kitagawa ${ }^{22}$, Geoffrey Manley ${ }^{23}$, Stephan Mayer ${ }^{24}$, David K. Menon ${ }^{25}$, Geert Meyfroidt ${ }^{26}$, Daniel B. Michael ${ }^{27}$, Mauro Oddo ${ }^{28}$, David Okonkwo ${ }^{29}$, Mayur Patel ${ }^{30}$, Claudia Robertson $^{31}$, Jeffrey V. Rosenfeld ${ }^{32,33}$, Andres M. Rubiano ${ }^{34,35}$, Juan Sahuquillo ${ }^{36}$, Franco Servadei ${ }^{37}$, Lori Shutter ${ }^{38}$, Deborah Stein ${ }^{39}$, Nino Stocchetti ${ }^{40,41}$, Fabio Silvio Taccone ${ }^{42}$, Shelly Timmons ${ }^{43}$, Eve Tsai ${ }^{44}$, Jamie S. Ullman ${ }^{45}$, Paul Vespa ${ }^{46,47,48,49}$, Walter Videtta ${ }^{50}$, David W. Wright ${ }^{51}$, Christopher Zammit ${ }^{52}$ and Randall M. Chesnut ${ }^{53,54,55,56^{*}}$ (D)

(0) 2019 The Author(s)

\begin{abstract}
Background: Management algorithms for adult severe traumatic brain injury (sTBI) were omitted in later editions of the Brain Trauma Foundation's sTBI Management Guidelines, as they were not evidence-based.

Methods: We used a Delphi-method-based consensus approach to address management of sTBI patients undergoing intracranial pressure (ICP) monitoring. Forty-two experienced, clinically active sTBI specialists from six continents comprised the panel. Eight surveys iterated queries and comments. An in-person meeting included whole- and small-group discussions and blinded voting. Consensus required $80 \%$ agreement. We developed heatmaps based on a traffic-light model where panelists' decision tendencies were the focus of recommendations.

Results: We provide comprehensive algorithms for ICP-monitor-based adult sTBI management. Consensus established 18 interventions as fundamental and ten treatments not to be used. We provide a three-tier algorithm for treating elevated ICP. Treatments within a tier are considered empirically equivalent. Higher tiers involve higher risk therapies. Tiers 1,2, and 3 include 10, 4, and 3 interventions, respectively. We include inter-tier considerations, and recommendations for critical neuroworsening to assist the recognition and treatment of declining patients. Novel elements include guidance for autoregulation-based ICP treatment based on MAP Challenge results, and two heatmaps to guide (1) ICP-monitor removal and (2) consideration of sedation holidays for neurological examination.
\end{abstract}

Conclusions: Our modern and comprehensive sTBI-management protocol is designed to assist clinicians managing sTBI patients monitored with ICP-monitors alone. Consensus-based (class III evidence), it provides management

\footnotetext{
*Correspondence: chesnutr@uw.edu

${ }^{56}$ Harborview Medical Center, University of Washington, 325 Ninth Ave, Mailstop 359766, Seattle, WA 98104-2499, USA

Full author information is available at the end of the article
} 
recommendations based on combined expert opinion. It reflects neither a standard-of-care nor a substitute for thoughtful individualized management.

Keywords: Brain injury, Head trauma, Algorithm, Protocol, Consensus, Intracranial pressure, Tiers, Seattle, SIBICC

\section{Introduction}

The Brain Trauma Foundation (BTF) Guidelines for the Management of Severe Traumatic Brain Injury (sTBI) [1] have been widely incorporated into practice and associated with a reduction in mortality from sTBI [2]. The first two editions [1,3] included popular treatment algorithms. However, because different sTBI treatments have been studied in isolation, almost no evidence exists regarding their relative efficacy, sequential ordering or combination as would be required to create evidencebased treatment protocols [4]. Later editions thus recognized that these algorithms could not be based on published evidence and omitted them $[5,6]$, to the expressed disappointment of many readers. In response to algorithm requests and the paucity of high-quality evidence for sTBI management algorithms, we here use a consensus approach to develop a management algorithm for sTBI that will be useful across a variety of management environments, with the condition that the sTBI patient is admitted to an ICU with an intracranial pressure monitor in situ.

We present a series of consensus-based algorithms for adult sTBI management. Our consensus working group (CWG) established 18 interventions as fundamental to sTBI care and ten treatments not to be used. It also established a three-tier algorithm focused on treating elevated ICP wherein higher tiers involve therapies with higher risk. We suggest considerations to address when advancing from lower to higher tiers and recommendations for critical neuroworsening aimed at assisting the recognition, workup, and treatment of declining patients. Novel elements include guidance for autoregulation-based ICP treatment and the performance of MAP Challenges, as well as two sets of heatmaps to guide (1) consideration of sedation holidays to facilitate neurological examination and (2) ICP-monitor removal.

\section{Methods}

Forty-two physicians comprised the CWG for the Seattle Severe Traumatic Brain Injury Consensus Conference (SIBICC) algorithm. The panel included ten neurointensivists, 23 neurosurgeons, five neurologist/ neurointensivists, two trauma surgeons, and two emergency medicine specialists. We based panel selection on (a) $>10$ years clinical experience in sTBI; (b) current, active involvement in acute care management of sTBI patients; (c) representation of involved disciplines; (d) geographic diversity; and (e) ability to commit time to the algorithm development process. We calculated panel size based on logistic considerations. Panelists completed conflict of interest forms relevant to sTBI management. There were no conflicts mandating recusal of any participant. In advance of the meeting all participants were provided with electronic copies of all manuscripts taken as evidence for the 4th edition Brain Trauma Foundation Guidelines for the Management of Severe TBI organized by chapter.

The expressed focus of the effort was to design a management algorithm that would be acceptable to the panel and amenable to application in both neurological and general ICUs by physicians not specialized in neurointensive care. Prior to the meeting, the CWG completed eight webbased surveys (SurveyMonkey Inc., San Mateo, California, USA, www.surveymonkey.com) to determine the algorithm design and focus, explore definitions and thresholds, operationally define treatment modalities, evaluate acceptability and tier assignments of treatment modalities, etc. We combined voting results with panelists' comments to iterate these surveys to maximize consensus and define areas requiring focus at the in-person meeting.

Two special surveys addressed the timing of ICP monitor removal and the use of sedation holidays for examinations. Early voting using discrete-elements did not progress toward resolution of these multivariate issues, so they were assembled into matrices, each cell of which represented a concatenation of multiple individual clinical indicators. By combining individual panelist's responses and color-coding the results using a trafficlight model (red-yellow-green), we constructed heatmaps to guide decision-making. We modified the Marshall CT classification [7] of post-operative imaging for this exercise. The post-surgical CT scans of patients having an evacuated mass lesion (EML) were additionally classified as if they had not had surgery (i.e., DI I-IV) and this ranking was appended to the EML label (e.g., EML/DI IV) in the heatmaps. We did this to increase the sensitivity of post-operative images to indicators of intracranial hypertension for this exercise.

The in-person SIBICC meeting occurred from the 5th to 7th of April 2019 in Seattle, Washington, USA. We used anonymous electronic voting and vote analysis (Electronic Media Services Inc., Gig Harbor Washington, USA, www.electronicmeetingservices.com). Professional, independent non-physician moderators facilitated group 


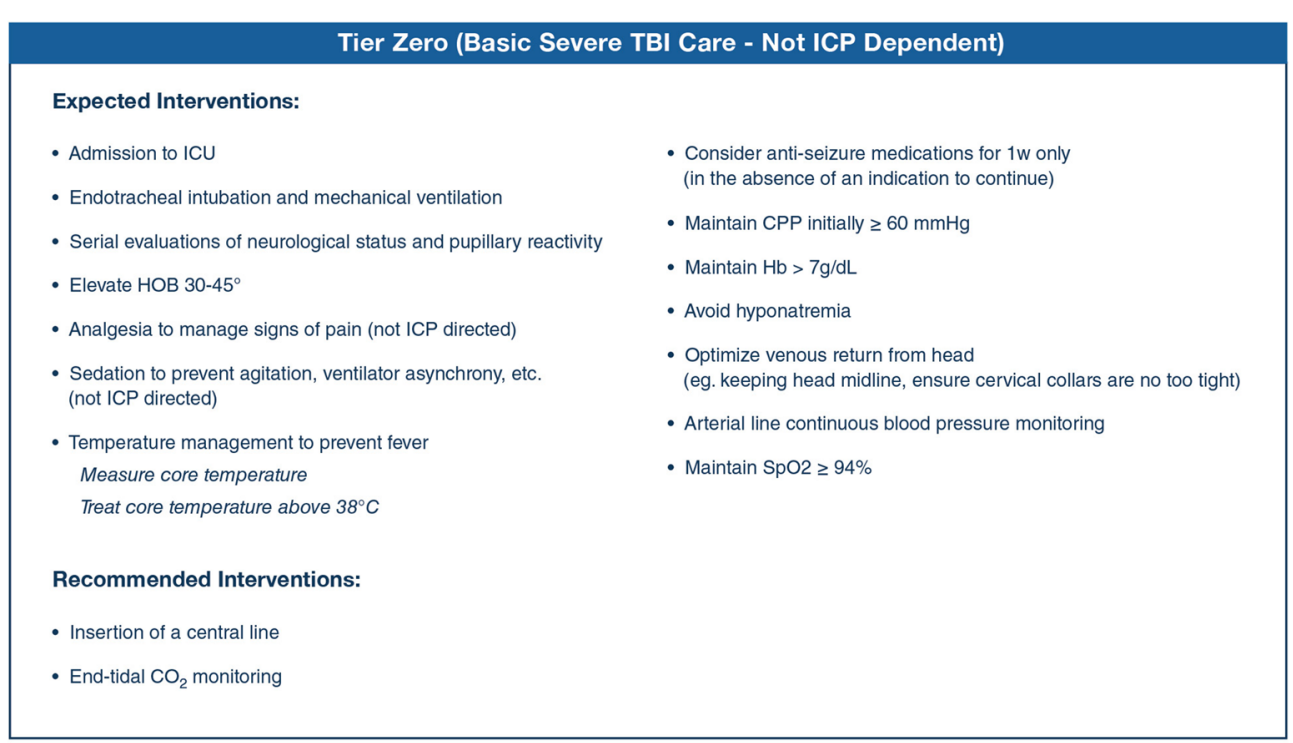

Fig. 1 Consensus-based basic severe traumatic brain injury care for patients with an ICP monitor in situ. These are basic treatments recommended as fundamental to the care of patients with sTBI, to be initiated ("Expected interventions") or considered ("Recommended interventions") upon ICU admission of a patient with an ICP monitor, regardless of the monitored pressure. $\mathrm{CO}_{2}$ carbon dioxide, $C P P$ cerebral perfusion pressure, $\mathrm{Hg}$ hemoglobin, $\mathrm{HOB}$ head of bed, ICP intracranial pressure, ICU intensive care unit, $\mathrm{spO}_{2}$ arterial oxygen saturation

discussions. We used small group sessions to address complex issues, with the whole CWG modifying and voting on small-group recommendations. All recommendations were incorporated verbatim into the final product. Unless specifically modified by the CWG, we limited the voting cycle to three iterations, interspersed with discussions. An element formed part of the final recommendations only if it achieved $80 \%$ agreement as "acceptable" (voting threshold) by at least $80 \%$ of the panelists (a quorum) in the confidential electronic voting. Unresolved issues are reported as such (Supplemental Material).

Donations solicited from industry and other interested parties funded this meeting. In return, they were allowed to silently observe the conference, without any interaction with the panelists or the process. No donors or other outside parties influenced any portion of these recommendations.

\section{Results}

Consensus-efforts generated a list of interventions viewed as fundamental to the care of sTBI patients and which should ideally be in place early in the course of care (Fig. 1). The CWG also generated a list of treatments that should not be used in the care of patients with severe TBI (Table 1), except in special circumstances. They developed a three-tier algorithm focused on the management of ICP elevation following severe TBI (Fig. 2). The CWG provided inter-tier recommendations (Fig. 2) and guidance on critical neuroworsening (Fig. 3) to assist in
Table 1 Treatment not recommended for use in the management of severe traumatic brain injury (when only ICP is monitored)

Mannitol by non-bolus continuous intravenous infusion

Scheduled infusion of hyperosmolar therapy (e.g., every 4-6 h)

Lumbar CSF drainage

Furosemide

Routine use of steroids

Routine use of therapeutic hypothermia to temperatures below $35^{\circ} \mathrm{C}$ due to systemic complications

High-dose propofol to attempt burst suppression

Routinely decreasing $\mathrm{P}_{\mathrm{a}} \mathrm{CO}_{2}$ below $30 \mathrm{mmHg} / 4.0 \mathrm{kPa}$

Routinely raising CPP above $90 \mathrm{mmHg}$

$C P P$ cerebral perfusion pressure, $I C P$ intracranial pressure, $\mathrm{kPa}$ kiloPascals, $\mathrm{P}_{a} \mathrm{CO}_{2}$ arterial partial pressure of carbon dioxide

evaluating and managing patients requiring increased therapeutic intensity. Heatmaps provide the CWG's consensus opinion on when it is safe to remove an ICP monitor (Fig. 4) and the safety of performing a sedationvacation aimed at obtaining an accurate neurological examination (Fig. 5).

\section{Discussion}

Sufficient research-based literature on individual ICP treatments exists to allow creation of an evidence report [6]. Unfortunately, there is insufficient research on the amalgamation of these individual treatments into a 


\section{Tier 1}

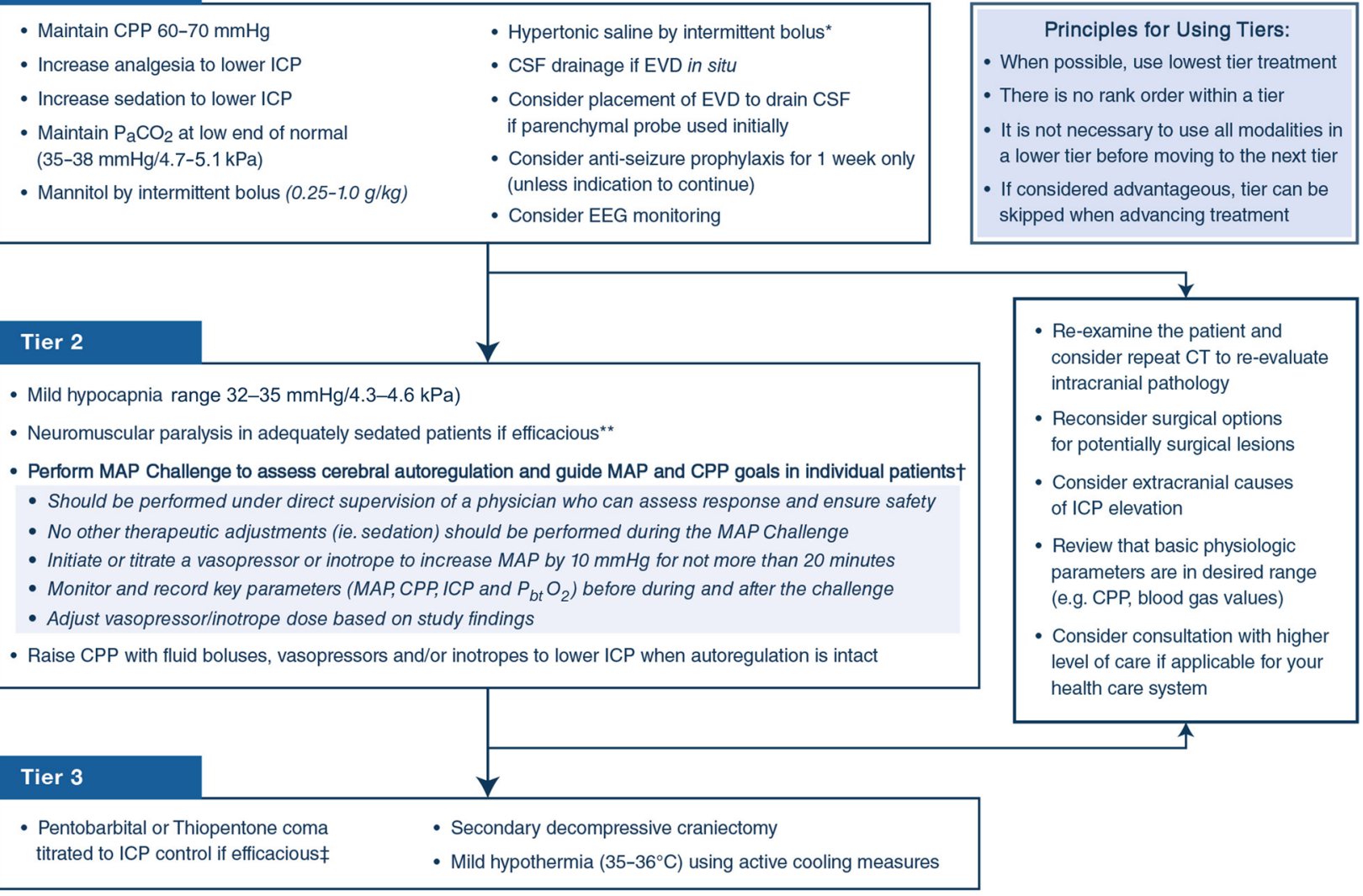

* We recommend using sodium and osmolality limits of $155 \mathrm{mEq} / \mathrm{L}$ and of $320 \mathrm{mEq} / \mathrm{L}$ respectively as administration limits for both mannitol and hypertonic saline.

** We recommend a trial dose of neuromuscular paralysis and only proceeding to a continuous infusion when efficacy is demonstrated.

$\dagger$ Rosenthal G. et al 2011

‡ Barbiturate administration should only be continued when a beneficial effect on ICP is demonstrated.

Titrate barbiturate to achieve ICP control but do not exceed the dose which achieves burst suppression.

Hypotension must be avoided when barbiturates are administered.

Fig. 2 Consensus-based algorithm for the management of severe traumatic brain injury guided by intracranial pressure measurements. Upper right box presents the principles for navigating through the treatments and tiers. Lower tier treatments are viewed as having a more favorable side effect profile than higher tiers and generally should be employed first. Inter-tier recommendations encourage patient reassessment for remediable causes of treatment resistance. See text for details. CPP cerebral perfusion pressure, EEG electroencephalogram, EVD external ventricular drain, ICP intracranial pressure, $\mathrm{KPa}$ kiloPascals, $\mathrm{MAP}$ mean arterial pressure, $P_{a} \mathrm{CO}_{2}$ arterial partial pressure of carbon dioxide

management algorithm to allow evidence-based development of a treatment approach. Since daily care consists of amalgamating individual treatments into a care plan, leaving this process to individual, non-expert practitioners without specific guidance is undesirable. Despite its limitations and weaknesses, the Delphi method remains the best current methodology for developing recommendations from expert consensus. As an evidentiary basis for prioritizing, sequencing, and combining sTBI treatments does not currently exist, we applied the Delphi method rigorously to this work, concomitantly recognizing the relative weakness of this approach.
Protracted specialist experience provides the expert practitioner repeated feedback on their manner of combining individual therapies. Although neither conditions nor outcomes are rigorously controlled, the impact of many repeated exposures to the influence of their therapeutic approach on short-term results (such as acute ICP control) should bestow a form of wisdom ("Medicine-Based Evidence"; the time-tested experience of an individual practitioner). An individual's wisdom is likely confounded by factors such as personal biases, technical idiosyncrasies, the care environment, patient population and injury demographics, etc. The concept of a consensus conference is to combine a large 


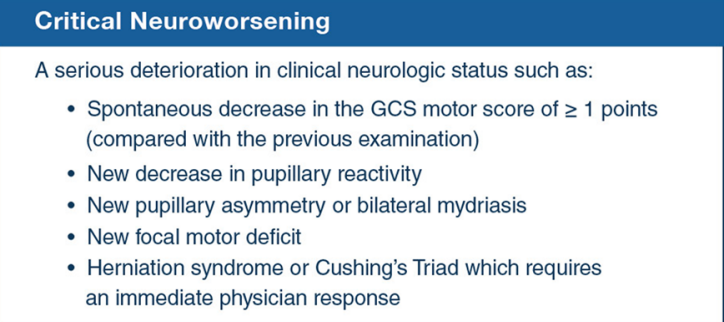

\section{Response to Critical Neuroworsening}

- Emergent evaluation to identify possible cause* of neuroworsening

- If herniation is suspected:

- empiric treatment

- hyperventilation ${ }^{\star *}$

- bolus of hypertonic solution

- consider emergent imaging or other testing

rapid escalation of treatment

${ }^{\star}$ Possible causes of neuroworsening include:

$\begin{array}{lll}\begin{array}{ll}\text { - expanding intracranial } \\ \text { mass lesion }\end{array} & \text { - medical comorbidity } & \text { - CNS infection } \\ \text { - cerebral edema } & \text { - medication effect } & \text { - infection or sepsis } \\ \text { - elevated ICP } & \text { hepatic function } & \text { - substance withdrawal } \\ \text { - stroke } & \text { - systemic hypotension } & \text { - dehydration } \\ \begin{array}{l}\text { - electrolyte or other } \\ \text { metabolic disturbance }\end{array} & \text { - seizure or post-ictal state } & \text { - hyper or hypothermia } \\ \text { ** the hyperemia/tissue hypoxia } & \end{array}$

Fig. 3 Critical neuroworsening and its management. SIBICC definition (upper box), response (middle box) and a list of suggested differential diagnoses (bottom) surrounding critical neurological deterioration (critical neuroworsening). CNS central nervous system, GCS Glasgow Coma Scale, ICP intracranial pressure

cohort of such experts and determine where their experiences overlap. As it is unlikely that individual-specific confounding factors will be shared, areas where there is general agreement should reflect a degree of clinical efficacy. The inclusion of multiple specialties, international sites, and varying practices should improve generalizability. We used this theoretical framework here to construct this set of recommendations.

Even with a large panel, blinded voting, and much discussion, such a process lacks rigor. As well, the observations on which the clinical wisdom is based primarily involve only ICP control and short-term clinical outcome. Therefore, the reader should consider these recommendations as a suggested treatment method without proven superiority over other applicable methods. Although primarily targeted at those with limited experience in monitor-based sTBI management, all practitioners involved in critical care treatment of sTBI patients may find these recommendations useful. They represent a safe and modern approach to sTBI care. They are not a standard of care or even the best treatment approach in a given instance. They are not legally binding. They are not designed as quality assurance monitoring tools. They do not represent the approach of any individual CWG member and should never be substituted for thoughtful clinical judgment. Hopefully, future scientific testing can increase the rigor of these recommendations and quantify their efficacy as steps toward establishing evidence-based algorithm development.

Derived by a large, international, multi-specialty consensus group through a rigorous Delphi-based consensus process, these recommendations are designed to augment other current management-related offerings such as the American College of Surgeons-Trauma Quality Improvement Program (ACS-TQIP) document [8], into which these recommendations are currently being integrated by the ACS Committee on Trauma (ACS-COT).

\section{Use of these recommendations in clinical practice Conditions of tiered treatment}

The use of tiers attempts to balance the benefits and efficacy of an agent against risks inherent to its use (Fig. 2). General clinical management is considered Tier Zero. Treatment of intracranial hypertension will generally begin at Tier-one. Movement to higher tiers reflects increasingly aggressive interventions. Treatments in any given tier are considered equivalent, with selection of one treatment over another based on individual patient characteristics and physician discretion. During any given episode being addressed, multiple

\footnotetext{
(See figure on next page.)

Fig. 4 Consensus views on the safety of intracranial pressure monitor removal in patients with acceptable ICP (no longer requiring active ICP management). The heatmap represents a summary analysis of the likelihood of each CWG member to remove the ICP monitor under differing conditions of stable pupillary status, GCS [20] motor score, modified CT classification (see "Methods"), duration of acceptable ICP, and degree of treatment previously required for any intracranial hypertension (none, Tier 1, or Tier 2 or 3). Green, yellow, and red indicate "safe to proceed", "consider proceeding with caution" and "do not proceed", respectively, with transitional shades reflecting intermediate trends. To use, choose the heatmap representing the ICP treatment history, then the appropriate status cell reflecting categorization of the patient in terms of the variables presented. The color in the relevant cell reflects the tendency of the CWG to withdraw the ICP monitor in that circumstance. It is up to the treating physician to consider the value of that tendency in making the final decision. AP abnormal pupils, CT computed tomography, DI diffuse injury as defined in the Marshall CT Head Score, GCS Glasgow Coma Scale, EML evacuated mass lesion as defined in the Marshall CT Head Score, ICP intracranial pressure, NP normal pupils
} 
Patients with NO intracranial hypertension since monitor insertion

\begin{tabular}{|c|c|c|c|c|c|c|c|c|c|}
\hline \multirow{2}{*}{\multicolumn{2}{|c|}{$\begin{array}{l}\text { NO INTRACRANIAL } \\
\text { HYPERTENSION }\end{array}$}} & \multicolumn{2}{|c|}{$\mathrm{GCS}_{\mathrm{M}} 6$} & \multicolumn{2}{|c|}{$\mathrm{GCS}_{\mathrm{M}} 5$} & \multicolumn{2}{|c|}{$\mathrm{GCS}_{\mathrm{m}} 4$} & \multicolumn{2}{|c|}{$\mathrm{GCS}_{M} 1-3$} \\
\hline & & NP & AP & NP & AP & NP & AP & $\mathrm{NP}$ & $\mathrm{AP}$ \\
\hline \multirow{4}{*}{$\begin{array}{c}\text { ICP } \\
\text { "acceptable" } \\
\text { for } 24 \text { hours }\end{array}$} & DI $1-2$ & & & & & & & & \\
\hline & EML / DI 1-2 & & & & & & & & \\
\hline & DI 3 & & & & & & & & \\
\hline & EML / DI III & & & & & & & & \\
\hline & & NP & AP & NP & AP & NP & AP & $\mathrm{NP}$ & $\mathrm{AP}$ \\
\hline \multirow{4}{*}{$\begin{array}{c}\text { ICP } \\
\text { "acceptable" } \\
\text { for } 48 \text { hours }\end{array}$} & DI $1-2$ & & & & & & & & \\
\hline & $\mathrm{EML} / \mathrm{DI} 1-2$ & & & & & & & & \\
\hline & DI 3 & & & & & & & & \\
\hline & EML / DI III & & & & & & & & \\
\hline & & NP & AP & NP & AP & NP & AP & NP & $\mathrm{AP}$ \\
\hline \multirow{4}{*}{$\begin{array}{c}\text { ICP } \\
\text { "acceptable" } \\
\text { for } 72 \text { hours }\end{array}$} & DI 1-2 & & & & & & & & \\
\hline & EML / DI 1-2 & & & & & & & & \\
\hline & DI 3 & & & & & & & & \\
\hline & EML / DI III & & & & & & & & \\
\hline & & NP & AP & NP & AP & NP & AP & $\mathrm{NP}$ & $\mathrm{AP}$ \\
\hline \multirow{4}{*}{$\begin{array}{c}\text { ICP } \\
\text { "acceptable" } \\
\text { for }>72 \\
\text { hours }\end{array}$} & DI 1-2 & & & & & & & & \\
\hline & EML / DI 1-2 & & & & & & & & \\
\hline & DI 3 & & & & & & & & \\
\hline & EML / DI III & & & & & & & & \\
\hline
\end{tabular}

Patients with intracranial hypertension requiring Tier 1 treatment - now controlled

\begin{tabular}{|c|c|c|c|c|c|c|c|c|c|}
\hline \multirow{2}{*}{\multicolumn{2}{|c|}{$\begin{array}{l}\text { MILD INTRACRANIAL } \\
\text { HYPERTENSION }\end{array}$}} & \multicolumn{2}{|c|}{$\mathrm{GCS}_{\mathrm{M}} 6$} & \multicolumn{2}{|c|}{$\mathrm{GCS}_{M} 5$} & \multicolumn{2}{|c|}{$\mathrm{GCS}_{\mathrm{M}} 4$} & \multicolumn{2}{|c|}{$\mathrm{GCS}_{\mathrm{M}} 1-3$} \\
\hline & & NP & AP & NP & AP & NP & AP & NP & AP \\
\hline \multirow{4}{*}{$\begin{array}{c}\text { ICP } \\
\text { "acceptable" } \\
\text { for } 24 \text { hours }\end{array}$} & DI 1-2 & & & & & & & & \\
\hline & EML / DI 1-2 & & & & & & & & \\
\hline & DI 3 & & & & & & & & \\
\hline & EML / DI III & & & & & & & & \\
\hline & & NP & AP & NP & AP & NP & AP & NP & AP \\
\hline \multirow{4}{*}{$\begin{array}{c}\text { ICP } \\
\text { "acceptable" } \\
\text { for } 48 \text { hours }\end{array}$} & DI 1-2 & & & & & & & & \\
\hline & EML / DI 1-2 & & & & & & & & \\
\hline & DI 3 & & & & & & & & \\
\hline & EML / DI III & & & & & & & & \\
\hline & & NP & AP & NP & AP & NP & AP & NP & AP \\
\hline \multirow{4}{*}{$\begin{array}{c}\text { ICP } \\
\text { "acceptable" } \\
\text { for } 72 \text { hours }\end{array}$} & DI 1-2 & & & & & & & & \\
\hline & EML / DI 1-2 & & & & & & & & \\
\hline & DI 3 & & & & & & & & \\
\hline & EML / DI III & & & & & & & & \\
\hline & & NP & AP & NP & AP & NP & AP & NP & AP \\
\hline \multirow{4}{*}{$\begin{array}{c}\text { ICP } \\
\text { "acceptable" } \\
\text { for }>72 \\
\text { hours }\end{array}$} & DI 1-2 & & & & & & & & \\
\hline & EML / DI 1-2 & & & & & & & & \\
\hline & DI 3 & & & & & & & & \\
\hline & EML / DI III & & & & & & & & \\
\hline
\end{tabular}

Patients with intracranial hypertension requiring Tier 2-3 treatment - now controlled

\begin{tabular}{|c|c|c|c|c|c|c|c|c|c|}
\hline \multirow{2}{*}{\multicolumn{2}{|c|}{$\begin{array}{c}\text { MODERATE-SEVERE } \\
\text { HYPERTENSION }\end{array}$}} & \multicolumn{2}{|c|}{$G C S_{M} 6$} & \multicolumn{2}{|c|}{$G C S_{M} 5$} & \multicolumn{2}{|c|}{$\mathrm{GCS}_{\mathrm{M}} 4$} & \multicolumn{2}{|c|}{$\operatorname{GCS}_{M} 1-3$} \\
\hline & & NP & AP & NP & AP & $\mathrm{NP}$ & AP & NP & AP \\
\hline \multirow{4}{*}{$\begin{array}{c}\text { ICP } \\
\text { "acceptable" } \\
\text { for } 24 \text { hours }\end{array}$} & DI 1-2 & & & & & & & & \\
\hline & EML / DI 1-2 & & & & & & & & \\
\hline & DI 3 & & & & & & & & \\
\hline & EML / DI III & & & & & & & & \\
\hline & & NP & AP & NP & AP & NP & AP & NP & AP \\
\hline \multirow{4}{*}{$\begin{array}{c}\text { ICP } \\
\text { "acceptable" } \\
\text { for } 48 \text { hours }\end{array}$} & DI 1-2 & & & & & & & & \\
\hline & EML / DI 1-2 & & & & & & & & \\
\hline & DI 3 & & & & & & & & \\
\hline & EML / DI III & & & & & & & & \\
\hline & & NP & AP & NP & AP & NP & AP & NP & AP \\
\hline \multirow{4}{*}{$\begin{array}{c}\text { ICP } \\
\text { "acceptable" } \\
\text { for } 72 \text { hours }\end{array}$} & DI $1-2$ & & & & & & & & \\
\hline & EML / DI 1-2 & & & & & & & & \\
\hline & DI 3 & & & & & & & & \\
\hline & EML / DI III & & & & & & & & \\
\hline & & NP & AP & NP & AP & NP & AP & NP & AP \\
\hline \multirow{4}{*}{$\begin{array}{c}\text { ICP } \\
\text { "acceptable" } \\
\text { for }>72 \\
\text { hours }\end{array}$} & DI 1-2 & & & & & & & & \\
\hline & EML / DI 1-2 & & & & & & & & \\
\hline & DI 3 & & & & & & & & \\
\hline & EML / DI III & & & & & & & & \\
\hline
\end{tabular}




\section{(See figure on next page.)}

Fig. 5 Consensus views on the safety of performing a sedation holiday aimed at obtaining an accurate neurological examination in patients whose ICP is controlled under different degrees of active management. The heatmap represents a summary analysis of the likelihood of each panelist to halt sedation to get an optimized neurological exam under differing conditions of stable pupillary status, GCS [20] motor score, modified CT classification (see "Methods"), duration of acceptable ICP with ongoing treatment, and degree of treatment previously required for any intracranial hypertension (none, Tier 1, or Tier 2 or 3). Green, yellow, and red indicate "safe to proceed", "consider proceeding with caution" and "do not proceed", respectively, with transitional shades reflecting intermediate trends. To use, choose the heatmap representing the ICP treatment history, then the appropriate status cell reflecting categorization of the patient in terms of the variables presented. The color in the relevant cell reflects the tendency of the CWG to perform a sedation holiday in that circumstance. It is up to the treating physician to consider the value of that tendency in making the final decision. AP abnormal pupils, CT computed tomography; D/ diffuse injury as defined in the Marshall CT Head Score, GCS Glasgow Coma Scale, EML evacuated mass lesion as defined in the Marshall CT Head Score, ICP intracranial pressure, NP normal pupils

items from a single tier can be trialed individually or in combination with the goal of a rapid response. The provider should maintain awareness of the duration of any episode and consider moving to more aggressive interventions in a higher tier quickly if the patient is not responding. In some cases, it might be preferrable to skip one or more tiers (e.g., choosing to decompress a patient with midline shift due to hemispheric swelling and very high initial ICP). No individual agent or combination thereof is critical to success in managing TBI. Clinical judgment must always determine the final management strategy.

\section{Tier-zero}

Tier-zero (Fig. 1) recommendations apply to sTBI patients with an ICP monitor in situ who are admitted to an ICU. Management recommendations for sTBI patients without ICP monitoring are published elsewhere [9]. The goal of Tier-zero is to establish a stable, neuroprotective physiologic baseline regardless of eventual ICP readings. Tier-zero sedatives and analgesics target comfort and ventilator tolerance rather than ICP. Temperature management targets avoiding fever (defined by our CWG as core temperature $>38{ }^{\circ} \mathrm{C}$ ). Consistent with the BTF Guidelines [6], the minimal cerebral perfusion pressure (CPP) threshold is $60 \mathrm{mmHg}$.

\section{Tier-one through tier-three (treatment of established intracranial hypertension)}

\section{Tier-one}

Tier-one (Fig. 2) represents the first foray into managing intracranial hypertension. The CWG adopted the BTF Guidelines' recommendations for initial ICP and cerebral perfusion pressure (CPP) thresholds $(22 \mathrm{mmHg}$ and $60 \mathrm{mmHg}$, respectively) [6]. Tier-one sedative or analgesic manipulation focuses on lowering ICP. The CWG decided not to recommend one hypertonic solution over the other, or to recognize different dosing levels (e.g., low and high dose) for either. After some discussion, they recommended using the same upper limits for serum sodium and osmolality for both agents.
When ICP is monitored using ventriculostomy, the CWG recommended CSF drainage as a Tier-one treatment. If an intraparenchymal device is used, consider ventriculostomy to enable CSF drainage. As subclinical seizure activity can cause intracranial hypertension, consider EEG monitoring and reconsider 1 week of prophylactic anti-epileptic drugs if not routinely used.

\section{Tier-two}

The hazards of routine neuromuscular blockade [10] prompted discussion regarding its position in Tiertwo versus three. The decision to place it at Tier-two includes a recommendation for basing continued administration on efficacy demonstrated during a trial period and to discontinue it as soon as possible.

Known risks of hyperventilation in sTBI [6] and frequent use of $\mathrm{P}_{b t} \mathrm{O}_{2}$ monitoring at many panelists' centres prompted caution in recommending even mild hyperventilation when brain oxygenation monitoring is not employed. To allow the maximum number of acceptable tools to those employing only ICP monitoring; however, the CWG recommended mild hyperventilation $\left(\mathrm{P}_{\mathrm{a}} \mathrm{CO}_{2} 32-35 \mathrm{mmHg} / 4.3-\right.$ $4.7 \mathrm{kPa}$ ) as a Tier-two treatment. They did not support lower $\mathrm{P}_{\mathrm{a}} \mathrm{CO}_{2}$ levels and recommended against routine hyperventilation to below $30 \mathrm{mmHg} / 4.0 \mathrm{kPa}$.

As increasing $\mathrm{CPP}$ can lower ICP if static pressure autoregulation (sPAR) is intact [11-13], the CWG recommended a simplified method of testing sPAR status based on that of Rosenthal et al. [14]. When CPP is above the lower breakpoint of sPAR and is then raised, the vasoconstriction attendant to active autoregulation decreases cerebral blood volume. This can be observed if the responses are trended, or by comparing the relevant parameters (i.e., ICP, MAP, and CPP) at the beginning and end of the test. As MAP elevation with disrupted SPAR may worsen intracranial hypertension, a practitioner capable of interpreting the results and halting the study should perform the test. If the trial is positive, judgment is required as to whether the benefit of the ICP decrease justifies the risks inherent to pharmacologically raising MAP. Cautious interpretation and application of the results of the MAP Challenge are recommended, as 

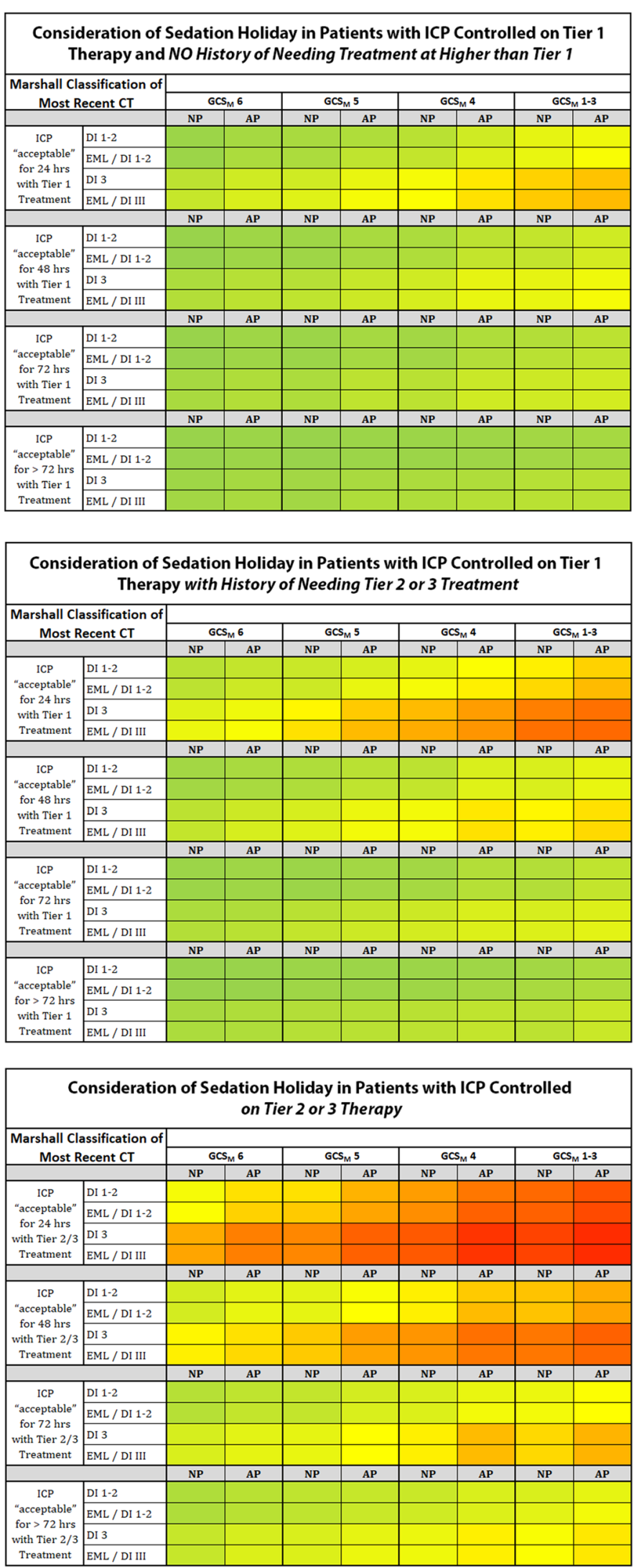
the test results may not be simple to interpret and the status of cerebral pressure autoregulation may not be stable.

The MAP Challenge is performed under stable conditions; to prevent confounding, no other active changes in care should be made during the challenge (including adjustments in sedation, analgesia, EVD drainage or other physiological parameters). To perform the challenge:

- Record baseline monitor parameters at the beginning of the challenge (e.g., ICP, MAP and CPP).

- Initiate or titrate a vasopressor to increase the MAP by $10 \mathrm{mmHg}$ for up to $20 \mathrm{~min}$.

- Observe the interaction between the MAP, ICP, and CPP during the challenge.

- Record monitor parameters at the end of the challenge.

- Evaluate the observed responses and recorded values for evidence of sPAR status. Disrupted sPAR will present as a sustained increase in ICP with MAP elevation.

- Adjust the target MAP back to baseline (disrupted SPAR) or to the chosen new, elevated target (intact SPAR).

\section{Tier-three}

Tier-three treatments have the greatest associated risks. The initiation of high-dose barbiturate therapy should be based on the response to a test dose. The CWG recommended EEG monitoring if barbiturates are continued and not titrating the dose upwards if burst suppression occurs, as further reduction in ICP is not anticipated and toxicity increases with dose. The endpoint of barbiturate treatment is ICP control not serum levels or EEG response. Hypotension must be avoided. The CWG supported use of mild hypothermia $\left(35-36{ }^{\circ} \mathrm{C}\right)$ but not routine therapeutic hypothermia to below $35{ }^{\circ} \mathrm{C}$ due to systemic complications. The CWG recommended decompressive craniectomy with recognition of the indications and hazards demonstrated by recent RCTs $[6,15$, $16]$.

\section{Not used}

The CWG recommended against the use of ten management items for treating established intracranial hypertension (Table $1 ; \geq 80 \%$ consensus). Some are specific pharmacologic agents or means of administering them. There are also specific physiologic parameters viewed as unsafe to exceed during treatment. The panel agreed that there may be circumstances where listed items might be reasonably used by an experienced and expert clinician in infrequent and carefully considered situations.
The CWG did not support hypothermia below $35^{\circ} \mathrm{C}$. Although hypothermia can lower ICP, there are no definitive outcome-based studies supporting recommendations. The evidence against its neuroprotective efficacy and demonstrated associated morbidity [17] were cited as reasons not to include it until studies on hypothermiamediated treatment of intracranial hypertension and recovery become available.

\section{Inter-tier recommendations}

Stepping to a higher tier is a potential indicator of increased disease severity. As higher tiers represent interventions with increased associated risks, the CWG recommends reassessing the patient's basic intra- and extra-cranial physiologic status and reconsidering the surgical status of intracranial mass lesions (e.g., contusions) not previously considered operative. If the patient is at a non-specialist centre at the point of upward tier advancement, the CWG recommended considering consultation with and potential transfer to a TBI center with increased resources if possible within the regional healthcare environment. When desired, transfer is best completed before clinical decline precludes it.

\section{Critical neuroworsening}

Critical neuroworsening represents a specific situation of critical deterioration requiring emergent evaluation and management. Neuroworsening was first defined retrospectively as a potential intermediate-outcome variable for TBI trials [18], it was adapted as a clinical variable for the BEST:TRIP trial [19] and subsequent management studies for limited resource environments [9]. The CWG adapted it here, terming the clinical definition "Critical Neuroworsening", to promote its recognition as a critical event and guide expeditious evaluation and consideration of empiric therapy.

\section{Weaning ICP treatment}

\section{Sedation holiday heatmaps}

The CWG recognized the balance between obtaining the most accurate (non-sedated) neurological examination of sTBI patients and the hazards of temporarily halting sedation to perform these examinations. Survey attempts at gaining consensus on relative and absolute contraindications were unsuccessful. The CWG therefore chose to construct matrices representing the most relevant decision variables under differing intracranial hypertension scenarios to recognize the distribution of the panel's opinions. The resulting heatmaps reflect the variability among expert clinicians in the perceived safety of sedation holidays in monitored sTBI patients. Using these decision-support matrices involves first determining which cell represents the patient in question. In situations 
where there are no other contraindications, green or "greenish" status supports sedation holiday use in line with the CWG's procedural recommendations. Red or "reddish" status represents relative recommendation against sedation holiday performance. The large areas appearing "yellowish" represent areas where the recommendations regarding proceeding are qualified, leaving to the clinician to decide if the current risk:benefit ratio might justify a sedation holiday. An alternative approach under "yellowish" conditions is to determine those factors that might be altered to shift the patient to a more highly recommended status. In all cases, however, the final decision is entirely up to the managing physician, with the heatmap serving only as an ancillary "consultation" from the CWG.

Procedural recommendations focus on minimizing risks and enhancing the utility of sedation holidays. Coordinating timing and optimizing the opportunity for all involved parties to be present (e.g., Intensivist, Neurosurgeons, etc.) maximizes the safety and interpretation of sedation holidays under all conditions. Finally, finding a favorable exam during the sedation holiday offers an opportunity to initiate sedation tapering.

\section{ICP monitor removal heatmap}

There is little literature regarding how long ICP should be acceptable before monitoring is discontinued. Marked variability in CWG survey results and lack of progression toward resolution over repeated surveys mimicked the sedation holiday conditions. Following the same logic, the CWG created heatmaps for ICP monitor removal. Using them for decision support again involves choosing the relevant heatmap and then the appropriate cell. The implications of the associated color parallel those described for the sedation holiday. Inspection of the heatmap trends confirms the marked variation among panelists. They suggest that $72 \mathrm{~h}$ of acceptable ICP is almost universally accepted whereas removal at $24 \mathrm{~h}$ is recommended only for patients with fairly benign CTs and favorable exams. The therapeutic intensity required prior to acceptable ICP and the Glasgow Coma Scale [20] motor score also appear to be strong influences on this decision. There is marked variability in the relative comfort with following the clinical examination versus continued monitoring in sTBI patients without continued intracranial hypertension. The value of the heatmap represents moderation rather than resolution of this controversy.

\section{Summary}

The goal of these SIBICC recommendations is to fill the void between the currently published evidence-based guidelines and clinical practice, using modern medicine-based-evidence to guide algorithm construction. Such algorithms provided a bridge between evidencebased analyses of individual treatments and the integration of these modalities into the management approach that is necessary at the bedside. The 42 clinically active global thought leaders with long-term experience in acute sTBI management who comprise the CWG used a Delphi-based consensus approach to produce a form of Medicine-Based-Evidence to facilitate the common situation of sTBI management at non-TBI centers. As consensus-based recommendations, they constitute the lowest level of evidence (Class III in the BTF Guidelines methodology [6]). Recognizing that these are recommendations-a form of multi-physician curbside consultthey are aimed at clinicians not specifically expert in the management of sTBI or those considering initiating ICP monitoring. They may be clinically adopted, adapted, referenced, or otherwise considered by individual physicians or medical centres. They may serve as useful tools for research design and should be subjected to analysis using comparative effectiveness research tools as well as trials. They are offered as guidance only, as a first attempt at filling a gap in the current clinical literature.

Electronic supplementary material

The online version of this article (https://doi.org/10.1007/s00134-019-05805-9) contains supplementary material, which is available to authorized users.

\begin{abstract}
Abbreviations
AP: Abnormal pupils; BTF: Brain Trauma Foundation; CNS: Central nervous system; CPP: Cerebral perfusion pressure; CSF: Cerebrospinal fluid; CT: Computed tomography; DI: Diffuse injury as defined in the Marshall CT Head Score; EEG: Electroencephalogram; EML: Evacuated mass lesion as defined in the Marshall CT Head Score; EVD: External ventricular drain; GCS: Glasgow Coma Scale; Hg: Hemoglobin; HOB: Head of bed; ICE: Imaging and clinical examination; ICP: Intracranial pressure; ICU: Intensive care unit; kPa: KiloPascals; MAP: Mean arterial pressure; NP: Normal pupils; $\mathrm{P}_{2} \mathrm{CO}_{2}$ : Arterial partial pressure of carbon dioxide; $\mathrm{P}_{\mathrm{bt}} \mathrm{O}_{2}$ : Partial pressure of brain tissue oxygen; $\mathrm{spO}_{2}$ : Arterial oxygen saturation.
\end{abstract}

\section{Author details}

${ }^{1}$ Section of Neurosurgery, University of Manitoba, GB1, 820 Sherbrook Street, Winnipeg, MB R3A 1R9, Canada. ${ }^{2}$ Almirante Nef Naval Hospital, Valparaiso University, Viña Del Mar, Chile. ${ }^{3}$ Valparaiso University, Valparaiso, Chile.

${ }^{4}$ Department of Neurosurgery, Medical School and Szentágothai Research Centre, Ifúság Útja 20, Pécs 7624, Hungary. ${ }^{5}$ University of Pécs, Pécs, Hungary. ${ }^{6}$ Department of Surgery, Harborview Medical Center, University of Washington, 325 Ninth Ave, Seattle, WA 98104-2499, USA. ${ }^{7}$ School of Medicine and Surgery, University of Milan-Bicocca, Milan, Italy. ${ }^{8}$ Anaesthesia and Intensive Care, San Gerardo and Desio Hospitals, ASST-Monza, Monza, Italy. ${ }^{9}$ Intensive Care Medicine, Australian and New Zealand Intensive Care Research Centre, Monash University, Melbourne, Australia. ${ }^{10}$ Department of Intensive Care and Hyperbaric Medicine, The Alfred Hospital, Melbourne, VIC, Australia. ${ }^{11}$ Department of Neurology, Penn Presbyterian Medical Center, University of Pennsylvania Perelman School of Medicine, 51 North 39th Street, Philadelphia, PA 19104, USA. ${ }^{12}$ Department of Neurology, Washington University School of Medicine, St. Louis, USA. ${ }^{13}$ Department of Neurology, Barnes-Jewish Hospital, 1 Barnes Jewish Hospital Plaza Suite 10400, St. Louis, MO 63110, USA. ${ }^{14}$ Division of Neurosurgery and Neuroscience Institute, Groote Schuur Hospital, University of Cape Town, H53 Old Main Building, Main 
Road, Observatory 7925, South Africa. ${ }^{15}$ Department of Neurosurgery, Renji Hospital, Shanghai Institute of Head Trauma, Shanghai Jiaotong University School of Medicine, 1630 Dongfang Road, Shanghai 200127, China.

${ }^{16}$ Department of Neurology, Johns Hopkins University School of Medicine, 600 N. Wolfe Street, Phipps 455, Baltimore, MD 21287, USA. ${ }^{17}$ Department of Neurosurgery, Stanford Neuroscience Health Center, 213 Quarry Rd 4th Fl, MC 5958, Palo Alto, CA 94304, USA. ${ }^{18}$ Department of Neurosurgery, 300 Pasteur Drive, Room R205, Edward's Building, MC: 5327, Stanford, CA 94305, USA. ${ }^{19}$ Department of Neurological Surgery, School of Medicine, Case Western Reserve University, 11100 Euclid Avenue, 5042, Cleveland, OH 44106, USA. ${ }^{20}$ Division of Neurosurgery, Department of Clinical Neurosciences, Addenbrooke's Hospital, University of Cambridge and Cambridge Biomedical Campus, Cambridge CB20QQ, UK. ${ }^{21}$ Department of Neurological Sciences, Christian Medical College, Ida Scudder Road, Vellore, Tamil Nadu, India. ${ }^{22}$ Vivian L Smith Department of Neurosurgery, McGovern Medical School at UTHealth, 6400 Fannin St, Suite 2800, Houston, TX 77030, USA. ${ }^{23}$ Department of Neurosurgery, San Francisco General Hospital and Trauma Center, University of California San Francisco, 1001 Potrero Ave., Bldg 1, Room 101, San Francisco, CA 94110, USA. ${ }^{24}$ Department of Neurology, Henry Ford Hospital, 2799 W Grand Blvd, Neurology, K-11, Detroit, Ml 48202, USA. ${ }^{25}$ Division of Anaesthesia, Addenbrooke's Hospital, University of Cambridge and Addenbrooke's Hospital, Hills Road, Box 93, Cambridge CB2 0QQ, UK. ${ }^{26}$ Department and Laboratory of Intensive Care Medicine, University Hospitals Leuven and KU Leuven, Herestraat 49, Box 7003 63, 3000 Leuven, Belgium. ${ }^{27}$ Department of Neurosurgery, Beaumont Health, Michigan Head and Spine Institute, Oakland University William Beaumont School of Medicine, Southfield, MI, USA. ${ }^{28}$ Department of Intensive Care Medicine, Faculty of Biology and Medicine, CHUV-Lausanne University Hospital, University of Lausanne, Lausanne, Switzerland. ${ }^{29}$ Department of Neurosurgery, University of Pittsburgh Medical Center Presbyterian, Suite B-400 200 Lothrop Street, Pittsburgh, PA 15213, USA. ${ }^{30}$ Department of Surgery, Vanderbilt University Medical Center, 1211 21st Avenue South, 404 MAB, Nashville, TN 37212, USA. ${ }^{31}$ Department of Neurosurgery, Baylor College of Medicine, One Baylor Plaza, Houston, TX 77030, USA

32 Department of Neurosurgery, Alfred Hospital, Melbourne, Australia. ${ }^{33}$ Department of Surgery, Monash University, Melbourne, Australia. ${ }^{34}$ INUB/ MEDITECH Research Group, Neurosciences Institute, El Bosque University, Bogotá, Colombia. ${ }^{35}$ MEDITECH Foundation, Clinical Research, Calle 7-A \# 44-95, Cali 760036, Colombia. ${ }^{36}$ Department of Neurosurgery, University Hospital Vall d'Hebron, Barcelona, Spain. ${ }^{37}$ Department of Neurosurgery, Humanitas University and Research Hospital, Milan, Italy. ${ }^{38}$ Department of Critical Care Medicine, Neurology and Neurosurgery, University of Pittsburgh Medical Center, 3550 Terrace St, Room 646, Pittsburgh, PA 15261, USA. ${ }^{39}$ Department of Surgery, Zuckerberg San Francisco General Hospital and Trauma Center, University of California, 1001 Potrero Ave., Ward 3A, San Francisco, CA 94110, USA. ${ }^{40}$ Department of Physiopathology and Transplantation, Milan University, Milan, Italy. ${ }^{41}$ Neuroscience Intensive Care Unit, Fondazione IRCCS Cà Granda Ospedale Maggiore Policlinico, Milan, Italy. ${ }^{42}$ Department of Intensive Care, Hospital Erasme, Université Libre de Bruxelles (ULB), Brussels, Belgium. ${ }^{43}$ Department of Neurological Surgery, GH 5100 SNEU, Indianapolis, IN 46202, USA. ${ }^{44}$ Suruchi Bhargava Chair in Spinal Cord and Brain Regeneration Research, The Ottawa Hospital, Department of Surgery, Division of Neurosurgery, University of Ottawa, Civic Campus, 1053 Carling Avenue, Ottawa, ON K1Y 4E9, Canada. ${ }^{45}$ Department of Neurosurgery, Donald and Barbara Zucker School of Medicine at Hofstra/Northwell, North Shore University Hospital, 300 Community Drive, 9 Tower, Manhasset, NY, USA.

${ }^{46}$ Gary L. Brinderson Family Chair in Neurocritical Care, UCLA School of Medicine, Los Angeles, USA. ${ }^{47}$ Critical Care Medicine Research, UCLA School of Medicine, Santa Monica, USA. ${ }^{48}$ Neurosurgery and Neurology, UCLA School of Medicine, Santa Monica, USA. ${ }^{49}$ Neurocritical Care, Ronald Reagan UCLA Medical Center, Santa Monica, USA. ${ }^{50}$ Intensive Care, Posadas Hospital, Buenos Aires, Argentina. ${ }^{51}$ Department of Emergency Medicine, Emory University School of Medicine, 49 Jesse Hill Jr Dr, Atlanta, GA 30303, USA. 52 Department of Emergency Medicine, University of Rochester Medical Center, 265 Crittenden Blvd, Suite 2100, Box 655C, Rochester, NY 14642, USA. ${ }^{53}$ Department of Neurological Surgery, University of Washington, Mailstop 359766, 325 Ninth Ave, Seattle, WA 98104-2499, USA. ${ }^{54}$ Department of Orthopaedic Surgery, University of Washington, Mailstop 359766, 325 Ninth Ave, Seattle, WA 98104-2499, USA. ${ }^{55}$ School of Global Health, University of Washington, Mailstop 359766, 325 Ninth Ave, Seattle, WA 98104-2499, USA.

${ }^{56}$ Harborview Medical Center, University of Washington, 325 Ninth Ave, Mailstop 359766, Seattle, WA 98104-2499, USA.

\section{Acknowledgements}

We thank our moderators Kevin Dunne and Theodore Michalke, Jone Howard for our audience response system, Kelley Chaddock for extensive administrative support, Peter Hendrickson, PhD for managing our web-based surveys, $\mathrm{PJH}$ is supported by the NIHR (Research Professorship, Cambridge BRC and Global Health Research Group on Neurotrauma), and DKM is an Emeritus Senior Investigator of the National Institute of Health Research (UK). We thank our financial supporters who include Adler/Geirsch Attorney at Law, the American Association of Neurological Surgeons/Congress of Neurological Surgeons Section on Neurotrauma and Critical Care, Bard, the Brain Trauma Foundation, DePuy, Hemedex, Integra, the Neurointensive Care Section of the European Society of Intensive Care Medicine, Neurosurgical Society of Australasia, Medtronic, Moberg Research, Natus, Neuroptics, Raumedic, Sophysa, Stryker, and Zoll.

\section{Compliance with ethical standards}

\section{Conflicts of interest}

No conflicts of interest relevant to this project were identified amongst the authorship group.

\section{Open Access}

This article is distributed under the terms of the Creative Commons Attribution-NonCommercial 4.0 International License (http://creativecommons.org/ licenses/by-nc/4.0/, which permits any noncommercial use, distribution, and reproduction in any medium, provided you give appropriate credit to the original author(s) and the source, provide a link to the Creative Commons license, and indicate if changes were made.

\section{Publisher's Note}

Springer Nature remains neutral with regard to jurisdictional claims in published maps and institutional affiliations.

\section{Received: 19 July 2019 Accepted: 25 September 2019}

Published online: 28 October 2019

\section{References}

1. Bullock R, Chesnut RM, Clifton G, Ghajar J, Marion DW, Narayan RK, Newell DW, Pitts LH, Rosner MJ, Wilberger JW (1996) Guidelines for the management of severe head injury. Brain Trauma Foundation. Eur J Emerg Med 3:109-127

2. Gerber LM, Chiu YL, Carney N, Hartl R, Ghajar J (2013) Marked reduction in mortality in patients with severe traumatic brain injury. J Neurosurg 119:1583-1590

3. The Brain Trauma Foundation, The American Association of Neurological Surgeons (2000) The Joint section on neurotrauma and critical care. Critical pathway for the treatment of established intracranial hypertension. J Neurotrauma 17:537-538

4. Schwartz ML, Tator CH, Rowed DW, Reid SR, Meguro K, Andrews DF (1984) The University of Toronto head injury treatment study: a prospective, randomized comparison of pentobarbital and mannitol. Can J Neurol Sci 11:434-440

5. Brain Trauma F, American Association of Neurological S, Congress of Neurological S, Joint Section on N, Critical Care AC, Carney NA, Ghajar J (2007) Guidelines for the management of severe traumatic brain injury. Introduction. J Neurotrauma 24(Suppl 1):S1-S2

6. Carney N, Totten AM, O'Reilly C, Ullman JS, Hawryluk GW, Bell MJ, Bratton SL, Chesnut R, Harris OA, Kissoon N, Rubiano AM, Shutter L, Tasker RC, Vavilala MS, Wilberger J, Wright DW, Ghajar J (2017) Guidelines for the management of severe traumatic brain injury. Neurosurgery 80:6-15

7. Lubillo S, Bolanos J, Carreira L, Cardenosa J, Arroyo J, Manzano J (1999) Prognostic value of early computerized tomography scanning following craniotomy for traumatic hematoma. J Neurosurg 91:581-587

8. Trauma ACoSCo (2015) Best practices in the management of traumatic brain injury. In: Book best practices in the management of traumatic brain injury

9. Hendrickson P, Pridgeon J, Temkin NR, Videtta W, Petroni G, Lujan S, Guadagnoli N, Urbina Z, Pahnke PB, Godoy D, Pinero G, Lora FS, Aguilera S, 
Rubiano AM, Morejon CS, Jibaja M, Lopez H, Romero R, Dikmen S, Chaddock K, Chesnut RM (2018) Development of a severe traumatic brain injury consensus-based treatment protocol conference in Latin America. World Neurosurg 110:e952-e957

10. Hsiang JK, Chesnut RM, Crisp CB, Klauber MR, Blunt BA, Marshall LF (1994) Early, routine paralysis for intracranial pressure control in severe head injury: is it necessary? Crit Care Med 22:1471-1476

11. Rangel-Castilla L, Gasco J, Nauta HJ, Okonkwo DO, Robertson CS (2008) Cerebral pressure autoregulation in traumatic brain injury. Neurosurg Focus 25:E7

12. Rosner MJ (1995) Introduction to cerebral perfusion pressure management. Neurosurg Clin N Am 6:761-773

13. Lang EW, Chesnut RM (2000) A bedside method for investigating the integrity and critical thresholds of cerebral pressure autoregulation in severe traumatic brain injury patients. Br J Neurosurg 14:117-126

14. Rosenthal G, Sanchez-Mejia RO, Phan N, Hemphill JC 3rd, Martin C, Manley GT (2011) Incorporating a parenchymal thermal diffusion cerebral blood flow probe in bedside assessment of cerebral autoregulation and vasoreactivity in patients with severe traumatic brain injury. J Neurosurg 114:62-70

15. Cooper DJ, Rosenfeld JV, Murray L, Arabi YM, Davies AR, D'Urso P, Kossmann T, Ponsford J, Seppelt I, Reilly P, Wolfe R, Investigators DT, Australian, New Zealand Intensive Care Society Clinical Trials G (2011) Decompressive craniectomy in diffuse traumatic brain injury. N Engl J Med 364:1493-1502
16. Hutchinson PJ, Kolias AG, Timofeev IS, Corteen EA, Czosnyka M, Timothy J, Anderson I, Bulters DO, Belli A, Eynon CA, Wadley J, Mendelow AD, Mitchell PM, Wilson MH, Critchley G, Sahuquillo J, Unterberg A, Servadei F, Teasdale GM, Pickard JD, Menon DK, Murray GD, Kirkpatrick PJ, Collaborators RET (2016) Trial of decompressive craniectomy for traumatic intracranial hypertension. N Engl J Med 375:1119-1130

17. Cooper DJ, Nichol AD, Bailey M, Bernard S, Cameron PA, Pili-Floury S, Forbes A, Gantner D, Higgins AM, Huet O, Kasza J, Murray L, Newby L, Presneill JJ, Rashford S, Rosenfeld JV, Stephenson M, Vallance S, Varma D, Webb SAR, Trapani T, McArthur C, Investigators PT, the ACTG (2018) Effect of early sustained prophylactic hypothermia on neurologic outcomes among patients with severe traumatic brain injury: the POLAR randomized clinical trial. JAMA 320:2211-2220

18. Morris GF, Juul N, Marshall SB, Benedict B, Marshall LF (1998) Neurological deterioration as a potential alternative endpoint in human clinical trials of experimental pharmacological agents for treatment of severe traumatic brain injuries. Executive Committee of the International Selfotel Trial. Neurosurgery 43:1369-1372 (discussion 1372-1364)

19. Chesnut RM, Temkin N, Carney N, Dikmen S, Rondina C, Videtta W, Petroni G, Lujan S, Pridgeon J, Barber J, Machamer J, Chaddock K, Celix JM, Cherner M, Hendrix T, Global Neurotrauma Research G (2012) A trial of intracranial-pressure monitoring in traumatic brain injury. N Engl J Med 367:2471-2481

20. Teasdale G, Jennett B (1974) Assessment of coma and impaired consciousness. A practical scale. Lancet 2:81-84 